\title{
Velocidade da bola no chute no futsal: comparação entre garotos com diferentes níveis de desempenho e correlação de variáveis preditoras do desempenho
}

CDD. 20.ed. 796.023

796.331

http://dx.doi.org/10.1590/1807-55092015000300371

\author{
Vitor Luiz de ANDRADE* \\ Luiz Henrique Palucci VIEIRA**** \\ Bruno Luiz de Souza BEDO* \\ Reinaldo MACARI* \\ Fábio Pamplona MARIANO* \\ Cesar Tadashi NODA*** \\ Paulo Roberto Pereira SANTIAGO**** \\ *Faculdade de Medici- \\ na de Ribeirão Preto, \\ Universidade de São \\ Paulo. \\ **Escola de Educação \\ Física e Esporte de \\ Ribeirão Preto, Univer- \\ sidade de São Paulo. \\ ***Instituto de Biociên- \\ cias, Universidade Esta- \\ dual Paulista-Rio Claro.
}

\section{Resumo}

0 estudo objetivou comparar e verificar as possiveis correlações entre a velocidade da bola com a velocidade do pé, ângulo relativo do joelho e comprimento do último passo durante o chute entre garotos Praticantes e Não Praticantes do futsal. Participaram do estudo 14 garotos com idade entre 13 e 15 anos divididos em dois grupos, Praticantes (G1) e Não Praticantes (G2) de acordo com seu tempo de prática estruturada semanal na modalidade. Para a análise cinemática duas câmeras ajustadas a uma frequência de $120 \mathrm{~Hz}$, foram fixadas lateralmente ao movimento realizado e focalizando os marcadores fixados no membro inferior do chute. Cada participante executou 10 chutes simulando uma cobrança de tiro livre no futsal. Os dados tridimensionais do membro inferior de chute foram obtidos pelo "software" DVIDEOW e tratados em ambiente Matlab, para obtenção das variáveis cinemáticas de interesse: velocidade da bola ( $\mathrm{VE}_{\mathrm{BOLA}}$ ), velocidade do pé $\left(\mathrm{VEL}_{\mathrm{PE}}\right)$, ângulo de joelho $\left(\mathrm{ANG}_{\mathrm{JOЕH \textrm {HO }}}\right)$ e comprimento do último passo (CP). Todas as variáveis exibiram índices estatisticamente maiores em $\mathrm{G} 1$ se comparado a $\mathrm{G} 2$ e, além disso, não foram encontradas diferenças intertentativas na tarefa ( $p$ $<0,05)$. No $\mathrm{G} 1$ uma análise de regressão múltipla somente revelou influência na $\mathrm{VEL}_{\text {BoLA }}$ em $17 \%$ causada pelo CP. Além disso, no $\mathrm{G} 2$ nenhuma variável influenciou na $\mathrm{VEL}_{\mathrm{BOLA}}$. Pode-se concluir que as variáveis estudadas são diferentes entre praticantes e não praticantes de futsal com idade entre $13-15$, e que a VEL ${ }_{\text {BoLA }}$ pode ser ligeiramente influenciada pelo CP nesta faixa etária. Recomenda-se que treinadores monitorem periodicamente o desenvolvimento desta caracteristica do movimento, principalmente em jogadores novatos, como forma de identificar jogadores com diferentes niveis de desempenho no chute em um contexto de futsal.

Palavras-Chave: Biomecânica; Cinemática; Habilidades motoras; Futsal.

\section{Introdução}

O desempenho do chute é um dos fatores-chave para marcar gols no futsal ${ }^{1}$. O desempenho relacionado a fatores biomecânicos nos chutes de finalização no futsal e no futebol são amplamente estudados ${ }^{1-6}$. Em situação específica, o padrão de movimento no chute pode ser influenciado pela situação do jogo, sendo dessa forma um fenômeno variável ${ }^{7}$. Além disso, o corpo pode assumir posturas diferentes de acordo com o alvo e velocidade da bola ${ }^{4}$.
Estudos anteriormente realizados encontraram positivas correlaçóes entre a velocidade do pé imediatamente antes do momento do impacto com a bola na velocidade da bola imediatamente após o contato $^{8-10}$. Adicionalmente, o comportamento da velocidade da bola após um chute de força máxima do membro dominante e não dominante pode ser afetado principalmente pelas diferenças na velocidade do pé e na mecânica de colisão ${ }^{11}$. 
Em diferentes níveis de prática foram verificadas diferenças no desempenho do chute, em que aqueles com nível de desempenho superior (elite e profissionais vs. não elite e amadores) para a categoria adulta, obtiveram melhor desempenho no chute ${ }^{12-15}$. Embora as pesquisas relacionadas à técnica de chute no futebol sejam amplamente conclusivas e bem delineadas devido ao avanço da ciência do esporte, o futsal ainda é carente de estudos que possam caracterizar melhor as diferenças do futebol para o futsal.

NewelL ${ }^{16}$ sugeriu que os movimentos dos seres humanos surgem das interações do organismo, do ambiente no qual os movimentos ocorrem e da tarefa a ser executada (ver também HaYwood e GetChell $^{17}$ ). Nesse sentido, a ação de "chutar", enquanto uma habilidade motora, é essencialmente a mesma quando comparados os contextos do futsal ao do futebol. Entretanto, existem algumas pequenas diferenças tanto em relação as restrições ambientais quanto da própria tarefa entre estes dois jogos esportivos coletivos que podem potencialmente influenciar o padrão de movimento neste elemento técnico de jogo. O futsal apresenta menor número de jogadores em quadra, dimensóes da área de jogo e tempo total de jogo ${ }^{18}$, além das diferenças em relação a superfície em que é praticado (piso rígido), menor tamanho e peso da bola e menor tamanho do alvo $(\mathrm{gol})^{19}$ e o tipo de calçado utilizado. Em um jogo de futsal, os jogadores executam em média 11,7 $\pm 4,13$ chutes no gol e 15,2 $\pm 3,43$ chutes para fora do gol ${ }^{20}$ enquanto que no futebol os jogadores executam um número menor, entre 1,2 e $2,5 \pm 2,2$ chutes $^{21}$ no total. A distância média do gol em que ocorrem os chutes no futebol é de 21,02 $\pm 9,22 \mathrm{~m}^{22}$ enquanto que no futsal a zona de maior número de chutes em gol fica localizada a uma distância de $10 \mathrm{~m}$ da linha de fundo ${ }^{23}$. Especificamente, quando se trata da análise cinemática do chute, KeLLIS e KaTIS ${ }^{24}$ indicaram que jogadores de futebol diminuem a velocidade de aproximação bem como nas rotaçôes e velocidades articulares para execução de um chute com acurácia, enquanto que BARBIERI et al. ${ }^{25}$ evidenciaram que no futsal esse fenômeno não ocorre, sugerindo que os jogadores priorizam principalmente a velocidade da bola durante chutes nesta modalidade, o que está em contraste com os achados no futebol ${ }^{25}$. Por isso, alguns pontos críticos como: comprimento do passo na fase balanço, ângulo de joelho no momento do contato com a bola, velocidade do pé e velocidade da bola no chute ainda necessitam de mais estudos no futsal, principalmente em categorias de formação.
Pesquisas anteriores sobre o chute no futsal verificaram diferença nos padróes cinemáticos e de coordenaçáo para diferentes restriçóes da tarefa, como chute envolvendo bola parada e em movimento em jogadores amadores adultos ${ }^{1}$. Em jovens jogadores a assimetria dos membros dominante e não dominante de chute ${ }^{2} \mathrm{e}$ as diferenças para a cinemática do membro de suporte durante a realização do chute ${ }^{3}$ foram confirmadas nas investigaçôes feitas, além de que a fadiga muscular parece afetar de forma contundente a velocidade da bola no chute em jogadores de futsal sub- $15^{26}$.

Entretanto, para nosso conhecimento, ainda não foram investigadas as mudanças nos padróes cinemáticos de chute em decorrência da prática estruturada do futsal, comparando por exemplo indivíduos jovens praticantes e não praticantes da modalidade. Além disso, a influência das variáveis que cercam a velocidade da bola também não foi investigada para o futsal nestes diferentes níveis, em categorias de formação. Investigaçôes conduzidas com o futebol evidenciam que mudanças fundamentais ocorrem nos padrōes de coordenação durante o chute como resultado da prática ${ }^{27}$. Entretanto isso ainda permanece no plano inferencial para o futsal, ou seja, não existem evidências que confirmem essa premissa.

Desse modo, os objetivos da presente investigação sáo comparar o padrão cinemático das variáveis: velocidade da bola, velocidade do pé, comprimento do passo e ângulo relativo do joelho relacionado ao chute em indivíduos praticantes e náo praticantes na modalidade futsal e verificar as possíveis correlaçóes entre as variáveis de interesse. A hipótese do presente estudo é de que existem diferenças no padrão cinemático do chute entre indivíduos com prática regular e que não praticam a modalidade futsal e de forma singular o desempenho do chute seja superior no grupo de praticantes. Além disso, caso existam, as diferenças pontuais na mecânica de chute entre os grupos, muito provavelmente elas impactariam diretamente a qualidade do contato do pé com a bola, e desta forma, espera-se que existam correlações importantes entre as variáveis testadas com a velocidade da bola apenas no grupo de praticantes.

Acreditamos que com os resultados da presente investigação, poderá se ter um melhor direcionamento das atitudes no treinamento para indivíduos com menor prática estruturada na modalidade e compreender as relações entre as variáveis que cercam a velocidade da bola no momento do chute para o futsal. 


\section{Método}

\section{Participantes \\ e delineamento experimental}

Para o estudo, foram convidados por conveniência 14 participantes do sexo masculino com idade entre 13 e 15 anos. Antes de qualquer avaliação, foi solicitada a assinatura individual pelos responsáveis legais do Termo de Consentimento Livre e Esclarecido aprovado pelo Comitê de Ética em Pesquisa do Instituto de Biociências da UNESP de Rio Claro. Os grupos foram divididos de acordo com a prática de cada garoto. Os que treinavam regularmente ou que faziam parte de algum programa de treinamento estruturado com no mínimo três sessóes por semana foram selecionados para participar do grupo de praticantes (G1). No grupo de náo praticantes (G2), os indivíduos foram selecionados quando náo participavam em nenhum programa de treinamento estruturado na modalidade e que responderam não gostar de praticar futsal ou futebol. Nenhum dos participantes era profissional.

\section{Tarefa}

Com no mínimo 24 e no máximo 72 horas depois da divisão dos grupos, foi realizada a tarefa propriamente dita. Inicialmente todos foram submetidos a um aquecimento e alongamento padronizado pelo treinador e adequado a posterior atividade. Em seguida, foram fixados marcadores reflexivos no membro inferior de chute.

Para o teste de chute de finalização foi posicionado um alvo no centro da meta ${ }^{1}$ e foi solicitado que realizassem 10 chutes com a bola parada posicionada na marcação do tiro livre direto de 10 metros sem a barreira na segunda marca de pênalti (TL10m). Os participantes foram orientados por uma instrução verbal idêntica para todos participantes, que foi: "chutem com o dorso do pé com a máxima força no alvo" ${ }^{1,28}$. Entre cada tentativa o participante teve liberdade de decidir o tempo de descanso e se o mesmo era necessário. Os chutes foram filmados por duas câmeras posicionadas e posteriormente a uma análise das filmagens as variáveis de interesse foram selecionadas e analisadas estatisticamente.

\section{Aquisição das Imagens}

Foram utilizadas duas câmeras digitais de vídeo (JVC, GR-DVL9800u ${ }^{\circledR}$ ) para a aquisição das imagens da execução dos chutes, ambas foram fixadas em tripés posicionados lateralmente ao movimento realizado de forma a focalizar os marcadores no membro inferior do chute (FIGURA 1). A frequência de aquisição das imagens foi de $120 \mathrm{~Hz}$, "shutter" de 1/250, foco ajustado manualmente e balanço do branco regulado para ambientes externos com grande luminosidade.

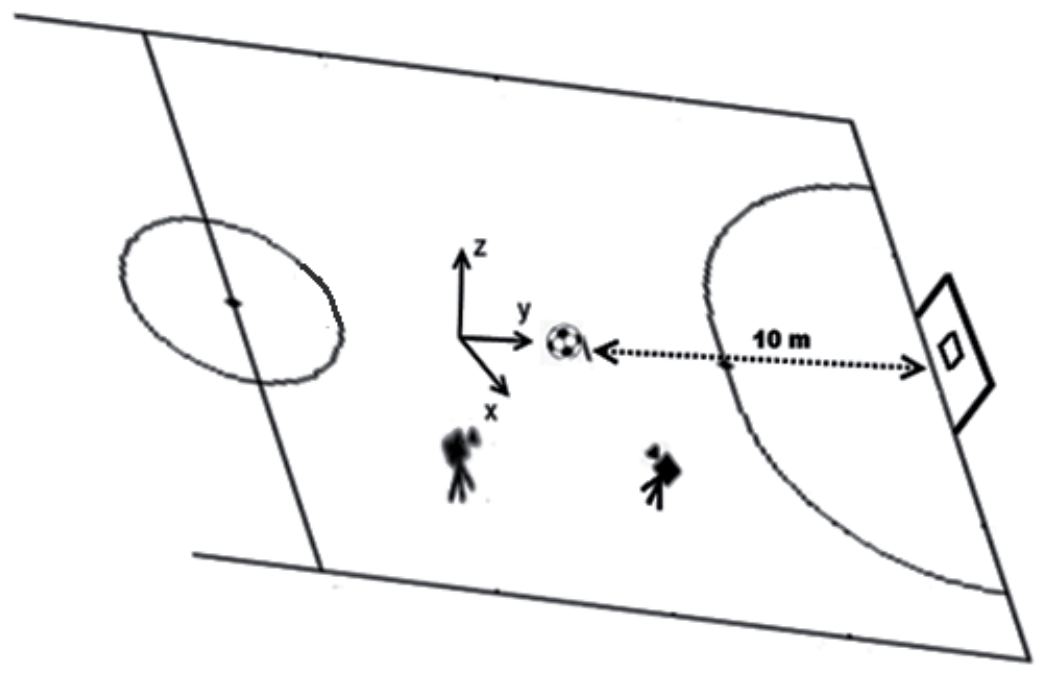

FIGURA 1 - Representação esquemática do posicionamento das câmeras para as coletas do TL10m.

\section{Aquisição das variáveis cinemáticas}

Previamente a realização dos chutes, foram fixados nos participantes marcadores reflexivos (esferas de 25 mm de diâmetro) nos seguintes pontos anatômicos: quadril (trocânter maior da cabeça do fêmur), joelho (epicôndilo lateral do fêmur), tornozelo (maléolo lateral), calcâneo (tuberosidade calcânea) e quinto 
metatarso (articulação metatarso falangeana do quinto metatarso). Os pontos representam os centros das articulaçóes de interesse para definir os segmentos coxa, perna e pé.

Para a aquisição das variáveis cinemáticas de interesse, foi utilizado o "software" DVIDEOW ${ }^{29-30}$ para o "desentrelaçamento", compactação, sincronização, medição e reconstrução tridimensional pelo método do DLT ("Direct linear transformation") ${ }^{31}$.

Para a reconstrução tridimensional foi utilizado um objeto calibrador contendo 12 marcadores com distâncias com base no sistema cartesiano de coordenadas previamente medidas. $\mathrm{O}$ eixo y foi definido na direção longitudinal da quadra de futsal no sentido do gol, o eixo x paralelo à linha de fundo da quadra com sentido para a linha lateral direita e ortogonal ao eixo y e o eixo $\mathrm{z}$ foi definido como produto vetorial de x por y (direção vertical apontando para cima) ${ }^{1,32}$.

Após este processo, foram obtidas matrizes de dados contendo as coordenadas espaciais de cada marcador medido em função do tempo $(x(t), y(t)$ e $\mathrm{z}(\mathrm{t})$ ), para cada chute realizado pelos participantes.

Para análise e tratamento dos dados cinemáticos foi utilizado o "software" Matlab". A velocidade tridimensional do pé e da bola foram calculadas pelo módulo do vetor de deslocamento do pé e da bola, antes e após o contato do pé com a bola.

$\mathrm{O}$ vetor deslocamento da bola $\left(\mathrm{VEL}_{\text {BOLA }}\right)$ foi calculado através das coordenadas da posição estacionária da bola (início), e no décimo quinto quadro após o contato do pé com a bola. $\mathrm{O}$ vetor de deslocamento do pé $\left(\mathrm{VEL}_{\mathrm{PE}}\right)$ foi calculado através do ponto médio entre os marcadores do calcâneo e do quinto metatarso, de três quadros de imagem que antecederam o contato do pé com a bola (início) até o contato do pé com a bola (final).

O ângulo relativo do joelho ( $\mathrm{ANG}_{\mathrm{JOELHO}}$ ) foi calculado através arco-coseno do produto interno entre os vetores normalizados da coxa e perna, no instante de

\section{Resultados}

As medidas cinemáticas do estudo obtiveram um erro de viés de 5,6 mm, uma precisão igual a 7,3 $\mathrm{mm}$ e a acurácia foi de $9,3 \mathrm{~mm}$. O erro de medida do estudo foi considerado aceitável, tendo em vista que é menor que o diâmetro dos marcadores utilizados nas avaliações $(25 \mathrm{~mm})$.

As variáveis cinemáticas $\mathrm{VEL}_{\mathrm{BOLA}}, \mathrm{VEL}_{\mathrm{PE}}$ ' $\mathrm{ANG}_{\text {JоELHO }}$ e CP foram significativamente diferentes contato do pé com a bola. Para o cálculo do comprimento do último passo (CP) foi utilizada a distância euclidiana das coordenadas tridimensionais da ponta do pé de chute no quadro que representa o momento em que o pé perde o contato com o solo até que atingisse as coordenadas tridimensionais do centro da bola.

\section{Determinação do erro de medida do estudo}

A determinação do erro de medida do estudo foi feito através de um teste específico proposto por EHARA et al. ${ }^{33}$ e também empregado por BARBIERI et al. ${ }^{1}$. Para isso, foi utilizada uma haste rígida contendo dois marcadores de $25 \mathrm{~mm}$ fixados nas extremidades a uma distância de 500 mm. Esta haste foi movimentada em torno de toda regiáo calibrada e a distância Euclidiana entre os dois marcadores durante o tempo foi obtida pelos mesmos procedimentos descritos anteriormente no tópico de "Aquisição das variáveis cinemáticas".

\section{Análise estatística}

A normalidade dos dados foi testada a partir do teste de Shapiro-Wilk. Verificada a normalidade, a análise descritiva foi apresentada pela média \pm desvio padrão. A análise de variância ANOVA "one-way" com análise "post-hoc" de Tukey foi utilizada em dois momentos, em um momento para verificar as diferenças entre os grupos (G1 e G2) nas variáveis $\mathrm{VEL}_{\text {BOLA, }}, \mathrm{VEL}_{\mathrm{PE}}, \mathrm{ANG}_{\text {JOELHO }}$ e CP, no segundo momento entre as tentativas das variáveis. Foi utilizada a análise de regressão múltipla para verificar o grau de influência das variáveis $\mathrm{VEL}_{\mathrm{PÉ}}, \mathrm{ANG}_{\mathrm{IOELHO}}$ e CP na $\mathrm{VEL}_{\text {BOLA }}$. Além disso, o teste de correlação de Pearson entre as tentativas em cada variável também foi feito. Em todos os casos o nível de significância foi pré-fixado em $\mathrm{p}<0,05$.

entre os grupos de Praticantes e Não Praticantes (TABELA 1).

Não foram encontradas diferenças intertentativas para as variáveis analisadas tanto para o grupo de Praticantes (G1) quanto para os Não Praticantes (G2) (FIGURA 2). 
TABELA 1 - Média (MD) £Desvio Padrão (DP) das variáveis provenientes dos chutes nos grupos com Praticantes (G1) e Não Praticantes (G2).

\begin{tabular}{lcccc}
\hline & \multicolumn{2}{c}{ G1 (n=7) } & \multicolumn{2}{c}{ G2 $(\mathbf{n}=7)$} \\
& MD & DP & MD & DP \\
\hline $\mathrm{VEL}_{\text {BOLA }}\left({\left.\mathrm{m} . s^{-1}\right)}^{-1}\right.$ & $17,15^{*}$ & 1,63 & 13,42 & 1,71 \\
$\mathrm{VEL}_{\text {PÉ }}\left(\mathrm{m} . \mathrm{s}^{-1}\right)$ & $14,87^{*}$ & 1,04 & 13,47 & 0,97 \\
$\mathrm{ANG}_{\text {JOELHO }}$ (graus) & $132,57^{*}$ & 14,23 & 125,50 & 11,84 \\
$\mathrm{CP}(\mathrm{m})$ & $1,02^{*}$ & 0,09 & 0,93 & 0,10 \\
\hline
\end{tabular}

*Indica diferença significativa em relação ao grupo G2. $p<0,05$.

G1

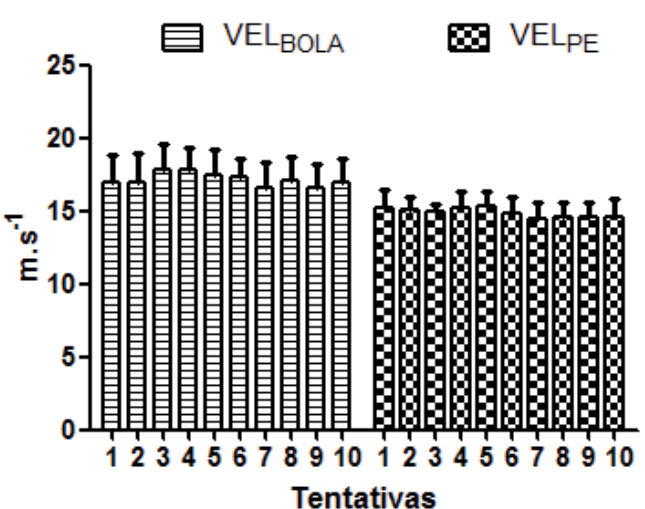

ANGJOELHO
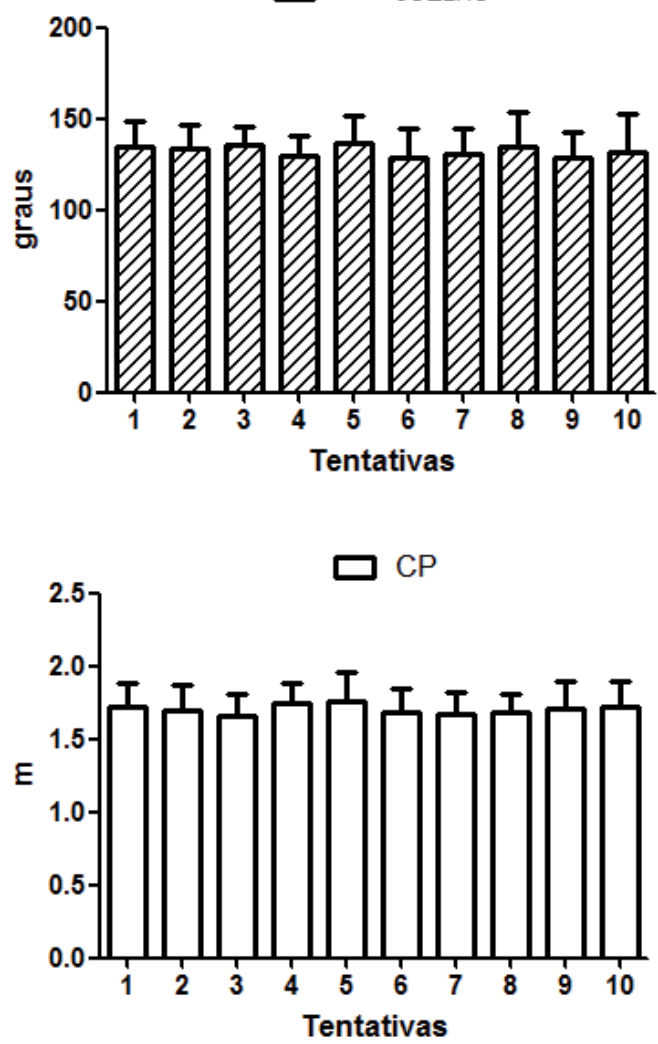

G2

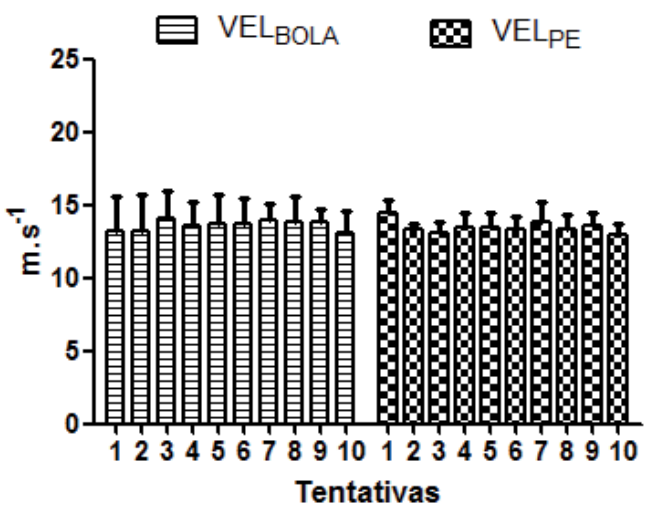

Z $\mathrm{ANG}_{\mathrm{JOELHO}}$
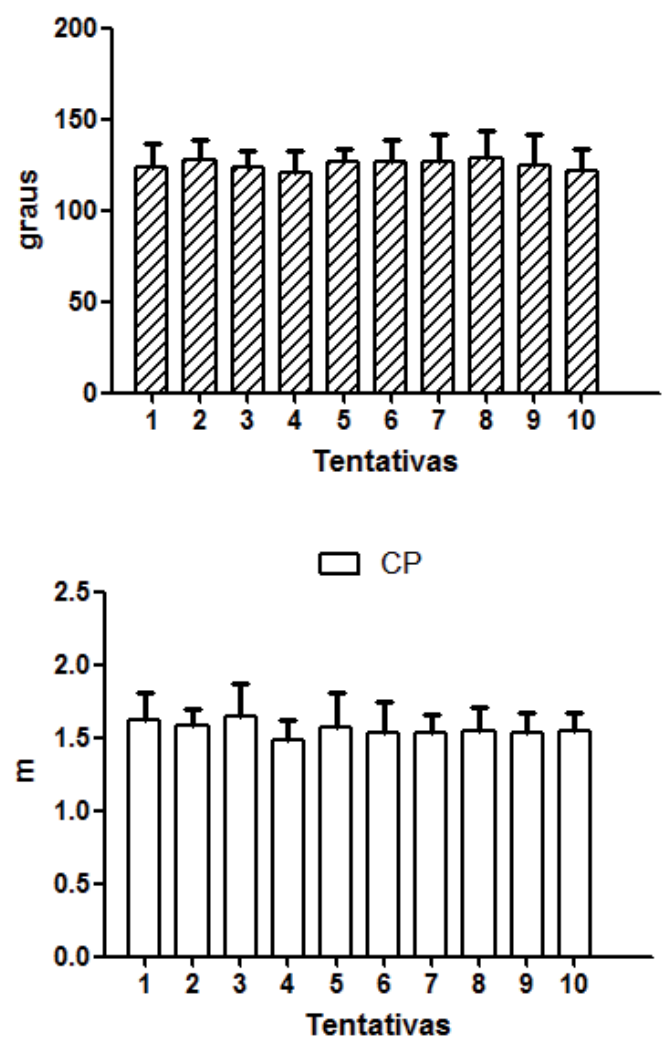

FIGURA 2 - Desempenho nas 10 tentativas dos sete participantes do G1 (Praticantes) e as 10 tentativas dos sete participantes do G2 (Não Praticantes). 
A análise de regressão múltipla para os dois grupos demonstrou um $\mathrm{R}^{2}$ fraco $(\mathrm{G} 1=0,31$ e $\mathrm{G} 2=0,04)$. No G1 a hipótese do estudo foi confirmada pelo teste $\mathrm{F}(\mathrm{F}=0,001)$ e foi encontrada uma influência positiva do CP de até $17 \%$ na $\mathrm{VEL}_{\text {BOLA }}(\mathrm{p}=0,01)$. No G2 a hipótese do estudo não foi confirmada (F $=0,35)$ e, além disso, não foi encontrada nenhuma influência das variáveis $\mathrm{VEL}_{\mathrm{PÉ}}, \mathrm{ANG}_{\text {JOELHO }}$ e CP sobre a $\mathrm{VEL}_{\text {BOLA }}$.

TABELA 2 - Coeficiente de correlação entre a VEL ${ }_{\text {BOLA }}$ e as variáveis VEL ${ }_{\mathrm{PE}}$, ANG $_{\text {JOELHO }}$ e CP em cada uma das tentativas no grupo Praticantes (G1).

${ }^{*} p<0,05$.

$* * p<0,01$

\begin{tabular}{|c|c|c|c|}
\hline & VEL $_{P E ́}$ & $\mathrm{ANG}_{\text {JOELHO }}$ & $\mathrm{CP}$ \\
\hline Tentativa 1 & 0,27 & 0,63 & 0,48 \\
\hline Tentativa 2 & $-0,01$ & $-0,36$ & 0,58 \\
\hline Tentativa 3 & $-0,11$ & 0,55 & 0,46 \\
\hline Tentativa 4 & 0,02 & 0,04 & $0,76^{*}$ \\
\hline Tentativa 5 & 0,61 & 0,19 & 0,43 \\
\hline Tentativa 6 & 0,11 & 0,03 & 0,37 \\
\hline Tentativa 7 & $-0,51$ & 0,63 & 0,71 \\
\hline Tentativa 8 & 0,26 & 0,62 & 0,63 \\
\hline Tentativa 9 & 0,30 & 0,48 & 0,03 \\
\hline Tentativa 10 & 0,44 & 0,49 & $0,88^{* *}$ \\
\hline
\end{tabular}

TABELA 3 - Coeficiente de correlação entre a VEL $\mathrm{BOLA}_{\mathrm{B}}$ e as variáveis $\mathrm{VEL}_{\mathrm{PE}}$, $\mathrm{ANG}_{\mathrm{JOELHO}}$ e CP em cada uma das tentativas no grupo Não Praticantes (G2).

${ }^{*} p<0,05$

\begin{tabular}{lccc}
\multicolumn{4}{c}{ tentativas no grupo Não Praticantes (G2). } \\
\hline & VEL $_{\mathbf{P E ́}}$ & ANG $_{\text {JOELHO }}$ & CP \\
\hline Tentativa 1 & $-0,56$ & $-0,36$ & $-0,60$ \\
Tentativa 2 & $-0,21$ & $-0,29$ & 0,43 \\
Tentativa 3 & $-0,25$ & $-0,79^{*}$ & $-0,39$ \\
Tentativa 4 & $0,78^{*}$ & $-0,49$ & 0,63 \\
Tentativa 5 & 0,27 & 0,28 & 0,43 \\
Tentativa 6 & 0,52 & 0,42 & 0,26 \\
Tentativa 7 & 0,22 & $-0,08$ & 0,44 \\
Tentativa 8 & 0,58 & $-0,23$ & 0,60 \\
Tentativa 9 & 0,17 & 0,04 & 0,04 \\
Tentativa 10 & 0,39 & 0,34 & 0,49 \\
\hline
\end{tabular}

\section{Discussão}

A presente investigaçáo verificou se as variáveis determinantes no chute de finalização do futsal eram diferentes de acordo com o nível de experiência do atleta e, se a $\mathrm{VEL}_{\mathrm{BOLA}}$ era dependente da $\mathrm{VEL}_{\mathrm{PÉ}}$, $\mathrm{ANG}_{\mathrm{JOelho}}$ e CP em jogadores Praticantes e Não
$\mathrm{AVEL}_{\text {BOLA }}$ no G1 foi significativamente correlacionada apenas nas tentativas 4 e 10 com o CP e nenhuma outra tentativa no $\mathrm{G} 1$ obteve correlação significativa entre a $\mathrm{VEL}_{\text {BOLA }}, \mathrm{VEL}_{\text {PÉ }}$ e $\mathrm{ANG}_{\text {JOELHO }}$ (TABELA 2).

No G2 a VEL ${ }_{B O L A}$ foi significativamente correlacionada apenas na quarta tentativa com a $\mathrm{VEL}_{\mathrm{PE}} \mathrm{e}$ negativamente correlacionada na tentativa 3 com o $\mathrm{ANG}_{\mathrm{JOELHO}}$ e nenhuma outra correlação significativa foi observada entre as tentativas nos grupos (TABELA 3).
Praticantes de futsal. Os principais achados foram que as variáveis determinantes para o chute de finalização no futsal são diferentes de acordo com o nível de prática na modalidade e, que a $\mathrm{VEL}_{\mathrm{BOLA}}$ não necessariamente é dependente das variáveis estudadas. 
A velocidade da bola média para G1 é bastante similar ao que foi verificado por BARBIERI et al. ${ }^{2}$ em jovens praticantes de futsal com idade similar ao participantes deste estudo na tarefa de TL10m $\left(18,2 \mathrm{~m} . \mathrm{s}^{-1}\right)$, e de jogadores titulares de equipes de elite sub-13 e sub-15 do Estado de Sáo Paulo (18,6 $\left.\mathrm{m} . \mathrm{s}^{-1}\right)^{34}$, e estấo acima do que foi verificado em jogadores sub-15 de uma equipe paranaense $(\sim 12$ $\left.\mathrm{m} . \mathrm{s}^{-1}\right)^{26}$. As equaçóes descritas na literatura para $\mathrm{o}$ futebol que explicitam a relação entre o vetor de $\mathrm{VEL}_{\text {BOLA }}$ com a VEL ${ }_{\mathrm{PE}}$ foram apresentadas e estão aproximadamente entre 1,06 e 1,23 $3^{35}$. No presente estudo a relação entre as variáveis foram de 1,15 para o G1 e 0,99 para o G2.

De acordo com Bernshteľ ${ }^{36}$ indivíduos pertencentes ao primeiro estágio de desenvolvimento de um comportamento habilidoso na execuçáo de tarefas tendem a simplificarem e reduzirem os movimentos nas articulaçôes envolvidas. Os valores inferiores de $\mathrm{ANG}_{\mathrm{JOELHO}}$ e CP em G2 representam menor amplitude de movimento nos participantes deste grupo, sobretudo na extensão da articulação do joelho durante o chute, e vão ao encontro do que propôs BernshteǏn ${ }^{36}$. A idade é um fator que interfere diretamente na mecânica do chute, e apresenta-se determinante pelo fato do aumento da força decorrente da maturação biológica ${ }^{37}$. Na presente investigação, a idade entre os grupos foi semelhante, entretanto, todas as variáveis entre si foram diferentes o que corrobora com os achados no futebol.

No presente estudo foram relatadas diferenças significativas para o desempenho do chute de finalização no futsal comparando-se grupos de indivíduos com diferentes níveis de desempenho dentro da modalidade, o que corrobora com achados da literatura específica para o futebol ${ }^{12-15}$ e reforça a ideia de que a prática induz mudanças nos padróes de coordenação ${ }^{27}$. ANDERSon e SidAWAY ${ }^{27}$ haviam reportado que novatos apresentam uma velocidade linear resultante do pé inferior a jogadores experientes no futebol. Estes autores haviam sugerido que isso ocorre por conta de fatores como uma menor amplitude de movimento do joelho em novatos. Tais fenômenos estão em acordo com nossos achados com o futsal, tendo em vista maiores índices de $\mathrm{VEL}_{\mathrm{PÉ}}$ e $\mathrm{ANG}_{\mathrm{JOELHO}}$ em G1 se comparado a G2.

AsAmi e NoLTE $^{38}$ encontraram forte correlaçấo entre $\mathrm{VEL}_{\mathrm{BOLA}}$ e $\mathrm{VEL}_{\mathrm{PE}} \mathrm{em}$ jogadores profissionais de futebol $(0,74)$, e BARBIERI et al. ${ }^{3}$, em estudo no futsal com jogadores amadores adultos, encontraram valores entre 0,67 - 0,76. Contudo, estudo recente de Sinclair et al..$^{39}$ utilizando uma análise de regressão múltipla não reportou significativa correlação entre $\mathrm{VEL}_{\text {BOLA }}$ e VEL ${ }_{\mathrm{PE}}$, quadro similar ao de LeEs et al. ${ }^{40}$ que também não encontraram correlação significativa entre tais variáveis no momento de impacto $(\mathrm{r}=$ 0,352), ambos no futebol. BARBIERI et al. ${ }^{2}$ também haviam relatado que a $V_{E L} L_{B O L A} \mathrm{eVEL}_{\mathrm{PE}}$ náo se correlacionam ( $r$ entre $0,15-0,42 ; p>0,05$ ) em jogadores praticantes de futsal com idade de 13,6 anos, o que está em acordo com os resultados em nosso estudo.

$\mathrm{Na}$ presente investigação os resultados mostram que há extensa possibilidade dos estudos referentes à $V L_{P E ́}$ no futebol não servirem como parâmetro para prediçáo da $\mathrm{VEL}_{\text {BOLA }}$ no futsal, algo que também foi evidenciado por BARBIERI et al. ${ }^{2}$. Uma das prováveis explicaçóes para isso é de que a bola de futsal pode apresentar um coeficiente de restituição menor se comparada a uma bola padronizada de futebol em virtude de suas características, como as dimensóes e a massa total. Por isso, há possibilidade, a partir dos resultados, de especular que a transferência de energia do quadril até a bola possa ser mais dissipada pela rigidez da bola no momento do impacto. Contudo, na presente investigação o coeficiente de restituição não foi determinado.

Outro parâmetro como $\mathrm{ANG}_{\mathrm{JOELHO}}$ está diretamente ligado ao desempenho no chute e representa a capacidade de transferência de energia da flexão do quadril, desaceleração da coxa e aceleraçáo da perna no momento do impacto com a bola. Nesse caso, a aceleração da perna em comum com a aceleração e velocidade final do pé gera uma maior $\mathrm{VEL}_{\text {BOLA }}{ }^{41}$. Entretanto, na presente investigação não foram encontradas quaisquer correlaçōes conclusivas ou influências na $\mathrm{VEL}_{\text {BOLA }}$ pelo $\mathrm{ANG}_{\text {IOELHO- }}$ O que reforça a hipótese do coeficiente de restituição da bola de futsal ser menor e, por isso, há uma perda maior de energia durante o impacto do pé com a bola. Dessa forma, sugere-se que futuras investigaçóes ocupem-se com esse fator, que possivelmente pode explicar as diferenças dos achados ente o futsal e o futebol.

A melhor representante do desempenho nos dois grupos foi o CP que caracteriza o afastamento da perna de chute e flexão de joelho na preparação da fase de balanço. O CP apresentou uma fraca influência na variável $\mathrm{VEL}_{\mathrm{BOLA}}(17 \%)$ para os atletas do G1, o que mostra a maior capacidade e experiência individual dos atletas do grupo para impor mais velocidade sobre a bola. Além disso, foram encontradas fortes correlaçóes $(r=0,76$ e $r$ $=0,88)$ em algumas tentativas no $\mathrm{G} 1$, ao contrário do G2 onde não foram evidenciadas correlaçôes significativas nem qualquer influência da medida 
na $\mathrm{VEL}_{\text {BOLA }}$. LeEs et al. ${ }^{40}$ evidenciaram correlação moderada $(r=0,419)$ entre o comprimento do último passo antes do chute e a velocidade da bola, em jogadores de futebol adultos. É provável que um maior comprimento do último passo antes do chute tenha feito com que os jogadores realizassem maior retração pélvica, fator que permitiu maior amplitude de movimento posterior na protraçáo pélvica durante a rotaçáo para frente do membro inferior de contato ${ }^{41}$. No entanto, nossos dados devem ser compreendidos com cautela, haja vista que as correlaçóes encontradas e a influência evidenciada nâo obtiveram valores satisfatórios de correlação na maioria das tentativas e o $\mathrm{R}^{2}$ da regressão múltipla não foi relevante.

Dois fatores relacionados ao chute poderão ser talvez interessantes para desenvolver um método que chegue mais próximo da real medida de predição da $\mathrm{VEL}_{\text {BOLA }}$ para o futsal. O primeiro estaria ligado à análise de mais variáveis relacionadas com o chute como a hiper flexão do joelho no momento da fase máxima de balanço e a flexão plantar. A segunda e provavelmente mais aceita estaria ligada ao desenvolvimento de uma equação de predição a partir da análise do chute em diferentes velocidades.

As principais limitaçóes do presente manuscrito são o baixo controle da amostra, haja vista que não foi controlada a idade dos indivíduos. Entretanto, a faixa de idade utilizada na amostra pode náo sofrer alteraçóes maturacionais que possam interferir nas análises ${ }^{42}$. A não mensuração da massa da perna e de alguns parâmetros importantes para a realização do chute como a flexão plantar e a flexão do joelho na fase de balanço e as poucas referências encontradas com essa temática para o futsal e a interpretação dos achados tomando como referência os achados anteriormente relatados no futebol tornam difíceis as conclusōes das reais contribuiçôes das variáveis estudadas para o desempenho do chute no futsal. Levando em consideraçáo que o futsal é um esporte adaptativo e complexo, sem uma sequência previsível de padrōes de ataque e defesa das equipes envolvidas no jogo ${ }^{43}$, mais estudos devem ser realizados cercando as variáveis para definir com acuidade as possíveis influências e correlações entre as variáveis testadas. Sobretudo, futuras investigaçôes deveriam investigar o desempenho do chute no futsal em um contexto que envolvesse as demandas técnicas, físicas e táticas de um jogo real, e não como uma habilidade motora "fechada" 34 .

Para que um professor possa ensinar e corrigir com sucesso a execução de um determinado fundamento é necessário que ele tenha conhecimento sobre algumas características do esporte e de seus praticantes (iniciantes ou alto nível) ${ }^{44}$. Desse modo, nós demonstramos que o comprimento do último passo parece ser um potencial preditor da velocidade da bola no futsal em jovens praticantes com idade entre 13 e 15 anos, o que até o momento não havia sido descrito. Desta forma, recomenda-se que treinadores monitorem periodicamente o desenvolvimento desta característica do movimento, principalmente em jogadores novatos. $\mathrm{O}$ chute é um movimento balístico, assim, gravaçóes de vídeo, mesmo que em baixa de frequência de aquisição e com uma simples câmera posicionada lateralmente durante a execução de chutes (i.e., em uma vista no plano sagital), certamente poderão auxiliar nesse processo, como é feito na aplicação do TGMD-2 por exemplo, que se baseia em avaliaçóes "offline" por meio de gravaçóes de vídeo ${ }^{45}$, sem o emprego de "softwares" para análises biomecânicas sofisticadas. Em adiçâo, sugere-se que futuros estudos desenvolvam escalas de desenvolvimento do chute no futsal, utilizando-se também de métodos de análise qualitativa, que até o momento não foram amplamente explorados para investigação desta e de outras habilidade neste esporte.

Portanto, de acordo com os resultados encontrados entre os dois grupos, pode-se concluir que as variáveis velocidade do pé, ângulo de joelho e o comprimento do passo (na fase de aproximação para o impacto com a bola) são diferentes entre indivíduos praticantes e não praticantes de futsal. Além disso, a $\mathrm{VEL}_{\text {BOLA }}$ pode ser ligeiramente influenciada pelo CP na faixa etária de 13 a 15 anos. Com isso, fica evidente a necessidade de novos estudos que se ocupem em identificar quais fatores que estariam relacionados ao desenvolvimento de alta velocidade da bola neste esporte, em crianças, adolescentes e também em profissionais de alto nível. 


\begin{abstract}
Ball velocity on the kick in futsal: comparison between youth with different performance levels and correlation of the predictive variables of performance

The aim of this study was to compare and verify the possible correlations between ball velocity with foot velocity, knee angle and length of last stride between practitioners and non-practitioners of futsal game. Fourteen young males with aged between 13 and 15 years old divided into two groups: practitioners $(\mathrm{G} 1)$ and non-practitioners $(\mathrm{G} 2)$, according with the time of futsal structured practice. For the kinematic analysis we had used two cameras at a sampling frequency of $120 \mathrm{~Hz}$, fixed laterally to the movement performed in order to focus the markers in the limb of kick. Each participant has performed ten kicks simulating a kick of ten meters in futsal. Three-dimensional data of the lower limb of kick were obtained in DVIDEOW software and treated in Matlab environment in order to obtain the variables of interest: ball velocity $\left(\mathrm{VEL}_{\mathrm{BAL}}\right)$, foot velocity $\left(\mathrm{VEL}_{\mathrm{FOOT}}\right)$, knee angle $\left(\mathrm{ANG}_{\mathrm{KNEE}}\right)$ and length of last stride (LLS). Statistically all data displayed higher levels in $\mathrm{G} 1$ compared to $\mathrm{G} 2$. In addition, no significant differences were found between trials in each group $(p<0.05)$. In $\mathrm{G} 1$ a multiple regression analysis revealed only influence on VEL $\mathrm{BALL}_{17 \%}$ caused by LLS. Moreover, in $\mathrm{G} 2$ any variable have influenced $\mathrm{VEL}_{\text {BALL }}$. However, we conclude that the variables are different between practitioners and non-practitioners of futsal game with aged between 13 and 15 years old, and the variable $\mathrm{VEL}_{\text {BALL }}$ could be little influenced by the LLS in this age band. Thus, we suggested that coaches maintain focus on development and monitoring this characteristic of movement, especially in novices, in order to identify players with different levels of performance of kicking in a futsal context.
\end{abstract}

KEY WoRds: Biomechanics; Kinematics; Motor skills; Futsal.

\title{
Referências
}

1. Barbieri FA, Gobbi LT, Santiago PR, Cunha SA. Performance comparisons of the kicking of stationary and rolling balls in a futsal context. Sports Biomech. 2010;9:1-15.

2. Barbieri FA, Gobbi LTB, Santiago PRP, Cunha SA. Diferenças entre o chute realizado com o membro dominante e não-dominante no futsal: variabilidade, velocidade linear das articulações, velocidade da bola e desempenho. Rev Bras Cienc Esporte. 2008;29:129-46.

3. Barbieri FA, Santiago PRP, Gobbi LTB, Cunha SA. Análise cinemática da variabilidade do membro de suporte dominante e não dominante durante o chute no futsal. Rev Port Cienc Desporto. 2008;8:68-76.

4. Katis A, Giannadakis E, Kannas T, Amiridis I, Kellis E, Lees A. Mechanisms that influence accuracy of the soccer kick. J Electromyogr Kines. 2013;23:125-31.

5. Scurr JC, Abbott V, Ball N. Quadriceps EMG muscle activation during accurate soccer instep kicking. J Sports Sci. 2011;29:247-51.

6. Nunome H, Asai T, Ikegami Y, Sakurai S. Three-dimensional kinetic analysis of side-foot and instep soccer kicks. Med Sci Sports Exerc. 2002;34:2028-36.

7. Ishii H, Yanagiya T, Naito H, Katamoto S, Maruyama T. Numerical study of ball behavior in side-foot soccer kick based on impact dynamic theory. J Biomech. 2009;42:2712-20.

8. Kawamoto R, Miyagi O, Ohashi J, Fukashiro S. Kinetic comparison of a side-foot soccer kick between experienced and inexperienced players. Sports Biomech. 2007;6:187-98.

9. Levanon J, Dapena J. Comparison of the kinematics of the full-instep and pass kicks in soccer. Med Sci Sports Exerc. 1998;30:917-27.

10. De Witt JK, Hinrichs RN. Mechanical factors associated with the development of high ball velocity during an instep soccer kick. Sports Biomech. 2012;11:382-90.

11. Dorge HC, Anderson TB, Sorensen H, Simonsen EB. Biomechanical differences in soccer kicking with the preferred and the non-preferred leg. J Sports Sci. 2002;20:293-9. 
12. Finnoff JT, Newcomer K, Laskowski ER. A valid and reliable method for measuring the kicking accuracy of soccer players. J Sci Med Sport. 2002;5:348-53.

13. Ali A, Williams C, Hulse M, et al. Reliability and validity of two tests of soccer skill. J Sports Sci. 2007;25:1461-70.

14. Coelho ESMJ, Figueiredo AJ, Simoes F, et al. Discrimination of u-14 soccer players by level and position. Int J Sports Med. 2010;31:790-6.

15. Russell M, Benton D, Kingsley M. Reliability and construct validity of soccer skills tests that measure passing, shooting, and dribbling. J Sports Sci. 2010;28:1399-408.

16. Newell KM. Constraints on the development of coordination. In: Wade MG, Whiting HTA, organizers. Motor development in children: aspects of coordination and control. Boston: Martinus Nijhoff; 1986. p.341-60.

17. Haywood KM, Getchell N. Desenvolvimento motor ao longo da vida. Porto Alegre: Artmed; 2010.

18. Gorostiaga EM, Llodio I, Ibanez J, et al. Differences in physical fitness among indoor and outdoor elite male soccer players. Eur J Appl Physiol. 2009;106:483-91.

19. Ré AN. Características do futebol e do futsal: implicaçóes para o treinamento de adolescentes e adultos jovens. Lect Educ Fís Deportes. 2008;127:483-91.

20. Dogramaci S, Watsford M, Murphy A. Changes in futsal activity profile in a multi-day tournament. J Sports Med Phys Fitness. 2014. Epub 2014 Oct 10. PubMed PMID: 25303071.

21. Carling C, Dupont G. Are declines in physical performance associated with a reduction in skill-related performance during professional match-play? J Sports Sci. 2011;29:63-71.

22. Moura F. Análise das açóes técnicas de jogadores e das estratégias de finalizaçóes no futebol, a partir do tracking computacional [dissertação]. Rio Claro (SP): Universidade Estadual Paulista, Instituto de Biociências; 2006.

23. Lapresa D, Alvarez L, Arana J, Garzón B, Caballero V. Observational analysis of the offensive sequences that ended in a shot by the winning team of the 2010 UEFA Futsal Championship. J Sports Sci. 2013;31:1731-9.

24. Kellis E, Katis A. Biomechanical characteristics and determinants of instep soccer kick. J Sports Sci Med. 2007;6:154-65.

25. Barbieri FA, Gobbi LT, Santiago PR, Cunha SA. Dominant-non-dominant asymmetry of kicking a stationary and rolling ball in a futsal context. J Sports Sci. 2015. Epub 2015 Jan 2. PubMed PMID: 25554927.

26. dos Santos AD. O efeito da fadiga nas variáveis cinemáticas do chute de jogadores de futsal sub-15 [Trabalho de Conclusão de Curso]. Londrina (PR): Universidade Estadual de Londrina; 2012.

27. Anderson DI, Sidaway B. Coordination changes associated with practice of a soccer kick. Res Q Exerc Sport. 1994;65:93-9.

28. van den Tillaar R, Ulvik A. Influence of instruction on velocity and accuracy in soccer kicking of experienced soccer players. J Mot Behav. 2014;46:287-91.

29. Barros RML, René B, Leite NJ, Figueroa P. Desenvolvimento e avaliação de um sistema para análise cinemática tridimensional de movimentos humanos. Rev Bras Eng Bioméd. 1999;15:79-86.

30. Figueroa PJ, Leite NJ, Barros RML. A flexible Software for tracking of markers used in human motion analysis. Comput Methods Programs Biomed. 2003;72:155-65.

31. Abdel-Aziz Y, Karara H. Direct Linear Trasnformation from comparator coordinates into object space coordinates in close-range photogrammetry: proceedings of the ASP/UI Symposium on Close-Range Photogrammetry. Urbana: American Society of Photogrammetrys; 1971. p.1-18.

32. Cunha SA, Barros RML, Lima Filho EC, Brenzikofer R. Methodology for graphical analysis of soccer kick using spherical coordinates of the lower limb. In: Spinks W, Reilly T, Murphy A, organizers. Science and soccer IV. London: Routledge; 2002, p.8-15.

33. Ehara Y, Fujimoto H, Miyazaki S, Mochimaru M, Tanaka S, Yamamoto S. Comparison of the performance of 3D camera systems .2. Gait Posture. 1997;5:251-5.

34. Ré AHN, Cattuzzo MT, Santos FMC, Monteiro CBM. Anthropometric characteristics, field test scored and match-related technical performance in youth indoor soccer players with different playing status. Int J Perform Anal Sport. 2014;14:482-92.

35. Reilly T, Williams AM. Science and soccer. Abingdon: Taylor \& Francis; 2003.

36. Bernshtĕn NA. The co-ordination and regulation of movements. Oxford: Pergamon, 1967.

37. Tol JL, Slim E, van Soest AJ, van Dijk CN. The relationship of the kicking action in soccer and anterior ankle impingement syndrome: a biomechanical analysis. Am J Sports Med. 2002;30:45-50.

38. Asami T, Nolte V. Analysis of powerful ball kicking. In: Matsui H, Kobayashi K, editors. Biomechanics VIII-B. Champaign: Human Kinetics; 1983. p.695-700. 
39. Sinclair J, Fewtrell D, Taylor PJ, Bottoms L, Atkins S, Hobbs SJ. Three-dimensional kinematic correlates of ball velocity during maximal instep soccer kicking in males. Eur J Sport Sci. 2014;14:799-805.

40. Lees A, Kershaw L, Moura F. The three-dimensional nature of the maximal instep kick in soccer. In: Reilly T, Cabri J, organizers. Science and football: the proceedings of the Fifth World Congress on Science and Football. London: Routledge; 2005, p.65-70.

41. Lees A, Asai T, Andersen TB, Nunome H, Sterzing T. The biomechanics of kicking in soccer: a review. J Sports Sci. 2010;28:805-17.

42. Degache F, Richard R, Edouard P, Oullion R, Calmels P. The relationship between muscle strength and physiological age: a cross-sectional study in boys aged from 11 to 15. Ann Phys Rehabil Med. 2010;53:180-8.

43. Corrêa UC, Alegre F, Freudenheim AM, Dos Santos S, Tani G. The game of futsal as an adaptive process. Nonlinear Dynamics Psychol Life Sci. 2012;16:185-203.

44. Cunha SA, Moura FA, Santiago PRP, Castellani RMP, Barbieri FA. Futebol: aspectos multidisciplinares para o ensino e treinamento. Rio de Janeiro: Guanabara Koogan; 2011.

45. Valentini NC. Validity and reliability of the TGMD-2 for Brazilian Children. J Mot Behav. 2012;44:275-80.

\section{Agradecimentos}

Os autores gostariam de direcionar agradecimentos a FAPESP [2000/07258-3 e 2013/08302-6] e a CAPES pelo suporte financeiro para a realização do presente estudo.

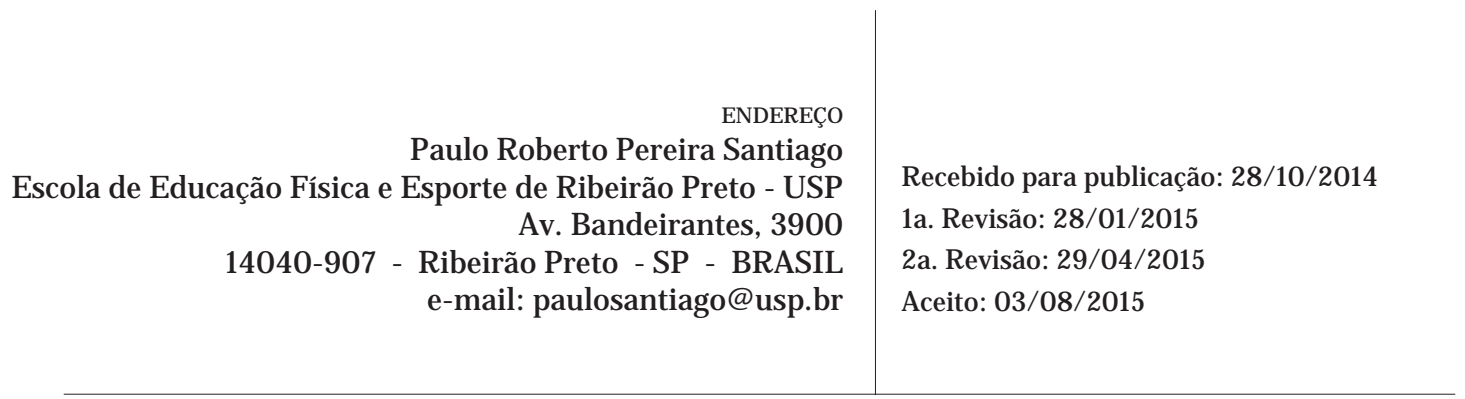

